\title{
Upgrade of the PAX H and D polarized internal target for precision measurements at COSY
}

\author{
G. Ciullo*; M. Statera, L. Barion, P. Lenisa. \\ INFN and University of Ferrara - I-44122 Ferrara (Italy) \\ E-mail: ciullo@fe.infn.it \\ G. Tagliente \\ INFN - Bari - I - 44122 Ferrara (Italy)
}

\section{A. Nass}

IKP-Jülich ForschungsZentrum Jülich - Germany

\begin{abstract}
The PAX Collaboration engaged the challenge to investigate the spin-dependendent cross-sections in order to exploit them for the polarization of antiproton beams. The commissioning of the tranverse case has been fullfilled on the proton COSY ring at the ForschungsZentrum of Jülich, and the results are milestones for the understanding of spin-dependent cross-sections. The whole panorama of spin-dependent interactions of proton beam on polarized proton target will be completed with the longitudinal case under preparation. Meanwhile the possibility of exploring the spin-filtering process with a deuteron polarized target, involves the upgrading of the PAX target for its daily switch from $\mathrm{H}$ to $\mathrm{D}$, and vice versa, for its use in same beam time period.
\end{abstract}

XVth International Workshop on Polarized Sources, Targets, and Polarimetry, September 9-13, 2013

Charlottesville, Virginia, USA

\footnotetext{
*Speaker.

$\dagger$ on behalf of the PAX collaboration
} 


\section{Introduction}

Facing the enourmous pontentialities of studying the interaction between polarized antiprotons $\left(\overline{\mathrm{p}}_{\uparrow}\right)$ and polarized protons $\left(\mathrm{p}_{\uparrow}\right)$, as an example the exclusive direct access to trasversity [1], still the main ingredient is missing: beams of polarized antiprotons, a challenging and promising tool capable of opening a new physics world.

The PAX collaboration, in the "antiproton proton scattering experiment with polarization" proposal [1], addressed the possibility of obtaining polarized antiproton beams via the interaction of stored unpolarized beams with polarized hydrogen atoms in an internal gaseous target, which acts like a polarization filter (spin-filtering).

In order to accomplish the missing tool, the PAX collaboration recently stimulated workshops [2,3], from which it came out that the only pursuable techinque is the spin-filtering process, which had shown its first experimental evidence on proton beams in 1992 [4].

The PAX collaboration, with its efforts to test and fully understand the spin-filtering process, already achieved important milestones $[5,6]$ in the storage ring COSY(COler SYnchrotron) at the ForschungsZentrum of Jülich(Germany). The program included the fulfillment of the the SPS CERN committee requirements for the approval of a proposal for the "Measurement of the Spin Dependence of the $\bar{p}$-p Interaction at the AD-ring" [7], which allows the test of the spin-filtering of $\overline{\mathrm{p}}$.

The understanding of the spin-dependent cross-sections beween unpolarized proton beam and polarized proton target at COSY is a viable way for the commissioning of the apparatus, for its exploitation for antiprotons at $\mathrm{AD}$ (Antiproton Decelerator) at CERN.

The spin-dependent cross-section studies and results, confirmed and clarified for the transverse case on proton [6], together with the possibility of polarized deuteron gaseous target, have excited theoretical studies on the extension of $\overline{\mathrm{p}}-\mathrm{p}_{\uparrow}$ interactions to the $\overline{\mathrm{p}}-\mathrm{d}_{\uparrow}$ interaction predictions. A large discrepancy in the estimation of the cross-sections and corresponding spin-filtering are reported [8], due to missing information on $\overline{\mathrm{p}}-\mathrm{p}_{\uparrow}$ spin dependent cross sections.

The efforts of the PAX collaboration to fulfill the complete scheme of interaction $\mathrm{p}-\mathrm{p}_{\uparrow}$, is itself challenging in extracting the spin dipendent cross sections, but also, it opens the possibility to explore the $\mathrm{p}-\mathrm{d}_{\uparrow}$ interaction, in the way to prepare the apparatus for proton and $\overline{\mathrm{p}}$ beams, together with a more ductile target of polarized proton and deuteron. The efforts, put by the collaboration on the project, pushes the COSY ring towards a well controlled and sharp machine for precision measurements.

\section{Spin-Filtering: how it works}

The interaction between a stored proton unpolarized beam on a polarized gaseous target can be explained starting from the physical quantity polarization $(P)$ :

$$
P=\frac{N_{\uparrow}-N_{\downarrow}}{N_{\uparrow}+N_{\downarrow}}
$$

where $N_{\uparrow}\left(N_{\downarrow}\right)$ is the population of particles with the same (opposite) spin orientation of a quantization axis. Polarization of particles of spin $1 / 2$ is defined by the equation (2.1). 
In order to distinguish between the polarization of the target and the stored beam, $Q$ indicates the target polarization, and $P$ that of the stored beam. The orientations of the spin are with respect to a quantization axis, which for the target is defined by its magnetic holding field. Instead for the beam in the COSY ring it is defined by the vertical axis, because in a circular accelerator, with vertical bending fields, the particle spin vector precesses around the vertical axis. Polarization is preserved along this axis.

The essential physical quantity for the $\mathrm{p}-\mathrm{p}_{\uparrow}$ interaction is the total cross section $\sigma_{t o t}$ of proton beam scattering on a polarized proton target:

$$
\sigma_{t o t}=\sigma_{0}+\sigma_{1}(\boldsymbol{P} \cdot \boldsymbol{Q})+\sigma_{2}(\boldsymbol{P} \cdot \boldsymbol{k})(\boldsymbol{Q} \cdot \boldsymbol{k}),
$$

where $\sigma_{0}$ is the spin-independent contribution to the total cross-section, $\sigma_{1}$ and $\sigma_{2}$ are the spindependent contributions, $\boldsymbol{Q}$ is the vector polarization of the target, $\boldsymbol{P}$ is the vector polarization of the beam, and $\boldsymbol{k}$ is the beam momentum versor.

Equation (2.2) provides two cases with respect to the target axis versor $\boldsymbol{q}=\boldsymbol{Q} / Q$ :

- transverse case: the quantization axis ot the target, therefore the target holding field is orthogonal to the momentum axis of the beam $\boldsymbol{k}$,

- longitudinal case : the quantization axis is parallel to the beam momentum.

For an initially equal populated state of spin 1/2 (at $\left.t=0: N_{\uparrow}=N_{\downarrow}\right) \sigma_{t o t}$ becomes respectively:

$$
\begin{array}{lll}
\boldsymbol{q} \cdot \boldsymbol{k}=0 & \text { (transverse) } & \sigma_{t o t \pm}=\sigma_{0} \pm \sigma_{1} Q, \\
\boldsymbol{q} \cdot \boldsymbol{k}=1 & \text { (longitudinal) } & \sigma_{\text {tot } \pm}=\sigma_{0} \pm\left(\sigma_{1}+\sigma_{2}\right) Q .
\end{array}
$$

The sign \pm in the equations (2.3) and (2.4) by convention is taken positive (negative) for same (opposite) orientation of the spin of the circulating beam with respect to $\boldsymbol{q}$. Particles of the beam with the same orientation of the target polarization are deflected less than the particles with opposite orientation of the spin (as a result $\sigma_{1}$ and $\sigma_{2}$ are negative). The unpolarized beam, in its repeated interaction with the polarized target, becomes polarized, with the same orientation of the target, but loses intensity.

The two spin-up and spin-down populations decrease, on each repeated interaction with the target, with different time constants. As a result, the polarization $P$ of the stored beam, following the equation (2.1) follows the time law: $P(t)=\tanh (t / \tau)$ [6].

Cross-sections have to be considered on the constraints of the ring, therefore "effective" $\sigma \mathrm{s}$ ( $\tilde{\sigma} s)$ have to used in the equations (2.3) and (2.4). The effective spin-dependent cross-section can be extracted from the time constant $\tau$ of the polarization build-up ( $d P / d t)[6]$ :

$$
\begin{gathered}
\text { transverse case } \frac{d P}{d t} \approx 1 / \tau_{\perp}=\tilde{\sigma}_{1} Q d_{t} f, \\
\text { longitudinal case } \frac{d P}{d t} \approx 1 / \tau_{\|}=\left(\tilde{\sigma}_{1}+\tilde{\sigma}_{2}\right) Q d_{t} f .
\end{gathered}
$$

Equations (2.5) and (2.6) contain the essential parameters for the studying of the spin-filter experiment: the target nuclear polarization $Q$, the thickness of the target $d_{t}$ (called also areal density), and the beam revolution frequency $f$. $\tilde{\sigma}$ indicates the effective cross section, taking into account 
that only particles scattered at an angle $\theta$ larger than the acceptance angle $\theta_{\text {acc }}$ contribute to the spin-filtering process: $\tilde{\sigma}=\sigma\left(\right.$ if $\left.\theta>\theta_{a c c}\right)$. In order to extract the more general physical quantities spin-dependent cross-sections from (2.5) and (2.6), the polarization of the beam $(P)$ has to be measured after the spin-filtering process has been performed.

\subsection{Beam polarization measurements}

After the spin-filtering process, the polarization of the beam is measured via $\mathrm{p}_{\uparrow}$ - $\mathrm{d}$ elastic scattering at the ANKE deuterium cluster jet target (fig. 1), by two Silicon Tracking Telescopes (STT), located left and right of the cluster ANKE target. The left-right asymmetry is measured at a beam energy, where the analyzing power is known. Details of the polarization measurements performed at COSY for the programs of the PAX experiments are reported in [5] and also in [6].

\section{Setup, results, and plans}

As a first attempt, the whole apparatus (polarized target, beam optics and accelerator devices) have been commissioned for the transverse case, due to the "natural" preservation of the transverse polarization in COSY, and for its direct and clear access to the spin-dependent cross section $\sigma_{1}$ as is clear from the equation (2.3).

The PAX collaboration employs all its effort in order to fullfil the requirement of the system for easily installation and operation on $\mathrm{AD}$ with antiproton. That means, once the apparatus are commissioned and the spin-filtering process is understood in the COSY ring, the whole PAX interaction point can be easily moved to the $\mathrm{AD}$ ring.

The confirmation of the spin filtering process and the extracted spin-dependent cross-section for the transverse case has been fulfilled in the 2011 [6].

The upgrading of the COSY ring for this purpose involved many parts and apparatus, requiring vacuum improvement along the whole ring, optics matching for low beam loss in the accelerator and at the PAX interaction point, and tuning and improved beam control, in order to avoid depolarization phenomena.

The next steps are completing the full panorama of the spin-dependent cross-section, commissioning the longitudinal case, and completing the whole program. This means that the PAX collaboration will have in hand the technique in order to accomplish investigation on antiprotons. The chance to have a polarized deuteron target, for the study of $\mathrm{p}(\overline{\mathrm{p}})-\mathrm{d}_{\uparrow}$ is included in the program.

\subsection{Transverse setup and results}

For the full understanding of the spin-filtering process, it is mandatory to access the physical quantities, which are responsible of the phenomenum, $\tilde{\sigma}_{1}$ and $\tilde{\sigma_{2}}$, with the constrains of the ring and the insertion of the accumulation cell of the target inside it.

The spin-filtering process required very stable conditions during the whole beam time. The improvement of the ring can be summarized in its increase of the lifetime from $800 \mathrm{~s}$ to $10000 \mathrm{~s}$, and the polarization lifetime greather than $2 \cdot 10^{5} \mathrm{~s}$, which is very high compared to the beam lifetime. The intensity of the beam for each filtering cycle were in average $5 \cdot 10^{9}$ particles/s. 


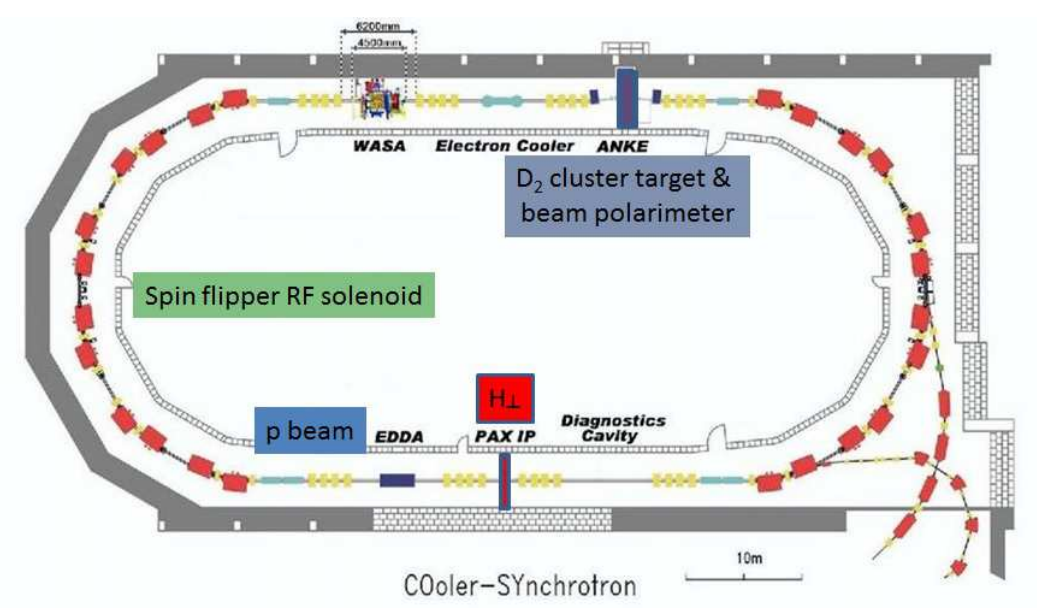

Figure 1: Scheme of the COSY ring. Main parts for the spin filtering are the $\mathrm{H}_{\perp}$ target at the PAX interaction point (IP) and the beam polarization measurements at the ANKE interaction point.

The target/filter of PAX, reported in figure 2 consists of a polarized Atomic Beam Source (pABS), an accumulation cell, which increases the integrated thickness of the target, and the dignostics system of a sampled gas from the center of the cell [12]. The cell was operated in a vertical magnetic field of 10 Gauss, limited to this low field in order to avoid orbit perturbations, which are compensated by two coils upstream and downstream from the target chamber. In a low target holding field one hyperfine state can be preserved and therefore injected, providing a measured target thickness of the cell $d_{t}=(5.5 \pm 0.2) \cdot 10^{13}$ atoms $/ \mathrm{cm}^{2}$ as expected. In the pABS for $\mathrm{H}$, the

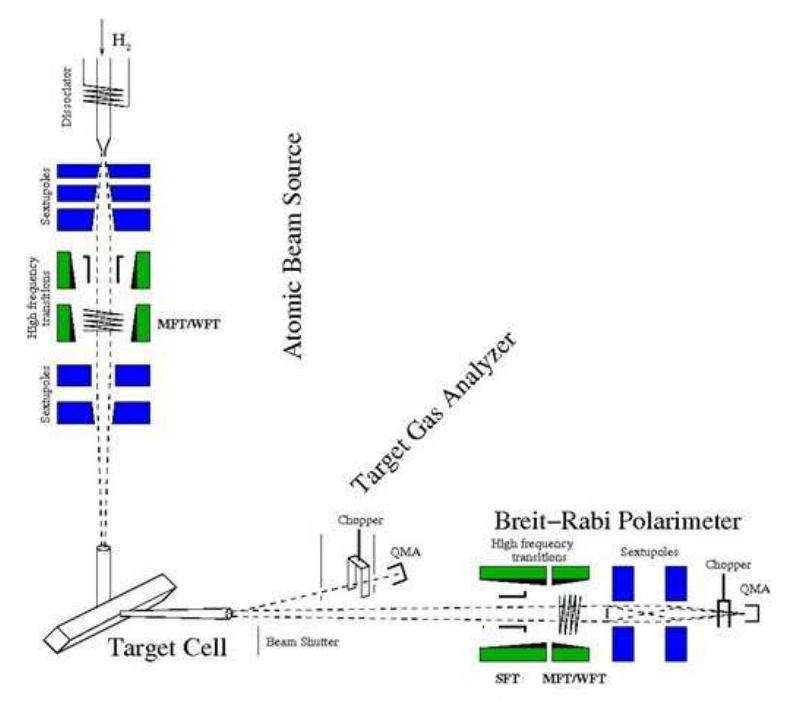

Figure 2: Scheme of the PAX target used as a filter. The pABS, the accumulation cell, and the dignostics devices: the TGA(Target Gas Analyzer) and the BRP(Breit-Rabi polarimeter.)

MFT is used during run and during the calibration of the BRP, injecting of state $\mid 1>$ (during run), and for the BRP calibration state $\mid 2>$ and both state also.

In the BRP we need to "scan" all the possible hyperfine states, in order to measure the polarization of the sampled gas from the center of the cell. Combinations of the MFT and SFT tran- 
sitions of the BRP allow measurements of the populations of each hyperfine state of the sampled gas $[7,11,12]$.

The perfomance of the target was very stable during the spin filtering time (run also until $16,000 \mathrm{~s})$. Different measured polarization after the spin-filtering process allows to provide a build-up of $d P / d t=(4.8 \pm 0.8) \cdot 10^{-7} \mathrm{~s}^{-1}$. With all the required physical quantities in the equation (2.5): $Q=0.73 \pm 0.05, d_{t}=(5.5 \pm 0.2) 10^{13}$ atoms $/ \mathrm{cm}^{2}, f=510032 \mathrm{~Hz}$ at the beam energy of $T_{p}=49.3 \mathrm{MeV}$, the measurement of spin dependent cross-section results $\tilde{\sigma}$ (meas) $=-$ $23.4 \pm 3.9$ (stat.) \pm 1.8 (syst.) $\mathrm{mb}$, fits well with the theoretical prediction, $\tilde{\sigma}$ (theor) $=-$ $26.9 \mathrm{mb}$, based on the present knowledge of the p-p spin-dependent interaction, as given in the SAID database, for the PAX -COSY constraints.

This result is the second one, at a different energy than [4], measurements of the spin-dependent cross-section for transverse case, which confirms the present understanding of the $\mathrm{p}^{\mathrm{p}} \mathrm{p}_{\uparrow}$ interaction, and it is a milestone for the spin-filtering process.

\subsection{Towards the longitudinal case}

For the longitudinal case, upgrades are required on the COSY ring for its use with longitudinally polarized beam. This can be accomplished in a straight section, opposite to that where a siberian snake will be installed [9]. A dedicated siberian snake has been designed for its installation at the ANKE interaction point (fig. 2), providing longitudinal polarized beams at the opposite straight section (where PAX interaction point is located). The PAX filter/target will be surrounded by a STT (Silicon Tracking Telescope) detector, covering a high detection angle, around the target, for spin observables. The detector is under design and construction following the experience and modules of ANKE and HERMES [14]. Polarization and other spin-observable measurements can be performed, meanwhile the spin-filtering is taking time.

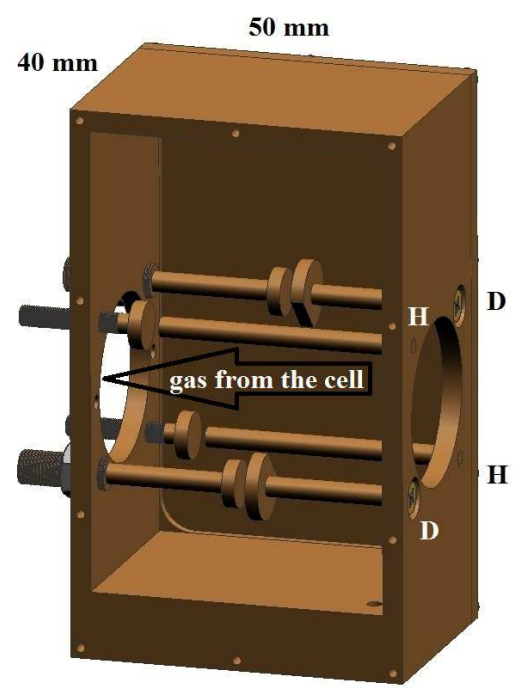

Figure 3: The SFT H\&D dual cavity. Coupled rods for $\mathrm{H}$ and $\mathrm{D}$ are tilted by $45^{\circ}$ with respect to the homogeneous and gradient field. than the zero-field hyperfine splitting frequency. The MFT transitions can be operated at a different frequency without exchanging them.

The polarized source commissioned for the transverse case, can be operated for the longitudinal case too, with the target magnetic field oriented along the beam momentum $\boldsymbol{k}$. The target chamber is already equipped for a $3 \mathrm{D}$ orientation of the magnetic field thanks to 3 sets of Helmholtz coils, installed on each face of the target chamber.

While COSY is upgrading for longitudinal polarized beam, the requirement of studying the spin filtering polarization by a vector polarized deuteron target pushes efforts to organize the target in a more ductile way.

\subsection{Extension to deuterium by a new dual cavity}

The whole system of the target to be switched from $\mathrm{H}$ to D requires a dedicated Strong field Transitions (SFT), resonating at a frequency a little higher 
In references $[11,10,12]$ more details are reported on the operation of the the pABS and the BRP, in its configuration for the HERMES experiment at DESY (Hamburg), and on its upgraded configuration for the transverse case at COSY (Jülich). The Ultra High Vacuum ( $10^{-10}$ mbar) of the diagnostic system (TGA and BRP in fig. 2) has to be broken, the SFT cavity has to be exchanged, and time is needed for vacuum chamber bake-out, tuning cavities and so on. As a result the change between $\mathrm{H}$ and $\mathrm{D}$, in both directions, takes usually weeks.

In order to have a possibilty to switch from $\mathrm{H}$ to $\mathrm{D}$, during a beam time experiment, a new cavity (fig. 3) was designed following the prototype developed at Ferrara [13] and tested for its resonance frequency, in order to obtain an oscillating magnetic field $\left(B_{R F}\right.$ at $45^{\circ}$ with respect to the homogeneous magnetic field of the cavity itself, allowing the $\sigma$ and $\pi$ transitions between hyperfine states).

For hydrogen in the pABS only the MFT transition is required. In the BRP the SFT - H (SFT for $\mathrm{H}$ ) and MFT are required, in order to scan on all the possible hyperfine populations. For the longitudinal case of $\mathrm{H}$ nothing will change. But, in order to investigate the possibility to switch forwards and backwards to $\mathrm{D}$, the dual cavity is already installed for its test first for $\mathrm{H}$ and then for D. The whole PAX target is under re-commissiong for $\mathrm{H}$ and $\mathrm{D}$ in a test area out of the COSY ring, meanwhile the upgrading program of the COSY ring for the longitudinal run is going on.

For Deuterium we need to put in operation for the pABS the standard SFT-D (this is a $\sigma$ cavity, that means the $B_{R F}$ oscillates parallel to the homogeneous field, allowing the $2 \leftrightarrow 6$ and $3 \leftrightarrow 5$, transitions for D). In the BRP we need to scan on all the possible states of D, therefore we will operate the SFT dual cavity for D ( $\sigma$ and $\pi$ cavity, this last allows the $1 \leftrightarrow 6,2 \leftrightarrow 5$, and $3 \leftrightarrow 6$ transitions) combined with a MFT transition.

\section{Conclusions}

The PAX collaboration measured the spin-dependent cross-section of a proton beam interacting with a polarized target of Hydrogen in the transverse case. The result agreed with the theoretical expectation. Future plans involve the upgrading of the COSY ring, for operating it with longitudinal polarized beam, and the design and construction, around the PAX cell, of a Si detector allowing a high coverage of detection angle for spin observables.

The target is straighforward for the longitudinal case of $\mathrm{H}$, but open questions on the $\overline{\mathrm{p}}-\mathrm{d}$ spin polarization cross-section, pushing the collaboration to prepare a daily switchable $\mathrm{H}$ or $\mathrm{D}$ polarized target/filter.

\section{References}

[1] V. Barone et al., "Antiproton-proton scattering experiments with polarization" arXiv:hep-ex/0505054 (2006) 1-200.

[2] "Polarized antiproton beams- How?" (Eds: S. Chattopadhyay et al.) AIP conf. Proc. 10082008.

[3] "Polarized Antiproton" WE-Heraeus-Seminar ( 23-25 june 2008 - Bad Honnef - Germany) http://www.fe.infn.it/heraeus/index.html.

[4] F. Rathmann et al., Phys. Rev. Lett. 7113791993.

[5] D: Oellers et al., Phys. Lett. B 674 (2009) 269-275. 
[6] W. Augustyniak et al., Phys. Lett. B 718 (2012) 64-69.

[7] C. Barschel et al, arXiv:0904.2325 [nucl-ex], Report number: CERN-SPSC-2009-012 ; SPSC-P-337.

[8] Yu N. Uzikov and J. Haidenbauer, Phis. Rev. C 87 (2013) 054003 [1-11] and their upgrading in Phys. Rev. C 88 (2013) 027001 [1-4].

[9] A. Lehrach (IKP): Accelerators COSY and HESR- NuPECC meeting "Nuclear physics in Jülich" 15-16 March 2013 Jülich - Germany.

[10] C. Baumgarten et al., NIMA 482 (2002) 606-618.

[11] A. Airapetian et al., NIMA 540 (2005) 68-101.

[12] G. Ciullo et al., J. Phys. Conf. Ser. 295 (2011) 012150.

[13] E. Steffens et al., in 20 ${ }^{\text {th }}$ Intern Symp. on Spin Physics (SPIN2012) september 17-22, 2012 JINR, Dubna, Russia.

[14] A. Airapetian et al., J. Instrum. 8 (2013) P05012. 